\title{
Commission 8: Astrometry
}

\author{
PRESIDENT: Imants Platais \\ VICE-PRESIDENT: Irina I. Kumkova \\ ORGANIZING COMMITTEE: E. Costa, C. Ducourant, \\ D. W. Evans, W.-J. Jin, M. G. Lattanzi, C.-L. Lu, R.-D. Scholz, \\ and M. Sôma
}

\begin{abstract}
This triennial report from Commission 8 covers astrometry-related matters for objects ranging from Solar system bodies out to Milky Way stars and QSOs at cosmological distances. This enormous range of distances is needed to establish, maintain, and improve the metric of the visible Universe-a very challenging effort since everything is moving. The progress of astrometry in the last three years (2002-2005) is reflected here. To locate the references, the reader is advised to check the NASA ADS Astronomy Abstract Service and the expanded report posted at URL http://www.pha.jhu.edu/iau_comm8/comm8.html
\end{abstract}

Keywords. Astrometry

\section{Scientific Highlights}

In this Report period, two large catalogues of positions and proper motions have been released and one is nearing the completion: the Lick Northern Proper Motion (NPM) by Hanson et al. (2004); the all-sky three-color and two-epoch USNO-B1.0 catalogue containing over one billion objects by Monet et al. (2003); and, the second release of the US Naval Observatory CCD Astrograph Catalog (UCAC2) by Zacharias et al. (2004). The first two catalogues crown and close the era of purely photographic sky surveys.

van Leeuwen has in essence single-handed re-analyzed the Hipparcos data aimed at a better understanding of the satellite's dynamics and its impact on derived astrometric parameters, also crucial to the Gaia success (van Leeuwen 2005).

Proper motion surveys combined with other techniques have led to the discovery of several low-mass "cool neighbors"-less than 10 pc away (Scholz et al. 2002, 2003, 2005)including the nearest brown dwarf $\varepsilon$ Indi B. This is an excellent example of synergy between astrometry and selected areas of astrophysics.

CHINA PR. Jin at Shanghai reports that the CCD camera (Model S1C-077) in drift scan mode was installed on the $1.56 \mathrm{~m}$ telescope of Shanghai Astronomical Observatory (SHAO). This work has been done by SHAO in collaboration with Nikolaev Astronomical Observatory (NAO), Ukraine. A collaborative project on joint observations and studies of NEOs, natural planetary satellites, binary and multiple stars is planned.

For CCD images with a small FOV, the Block Adjustment method was developed to achieve a precise astrometric calibration of multiple overlapping CCD images over a considerably larger area (Peng et al. 2004). The equations of block adjustment are given in the vectorial form. The Block Adjustment method was tested on simulated data as well as on real observations, which show that the overlapping CCD imaging can effectively improve the local reference frame and provide consistent results ( $\mathrm{Yu}$ et al. 2004).

JAPAN. Sôma at Mitaka reports on new formulae for precession and the relationship among UT1, GAST, and ERA developed to meet the high precision demands in VLBI observations (Fukushima 2003, 2004, Harada \& Fukushima 2004). 
A new method has been developed to obtain simultaneously from eclipse records the lunar tidal acceleration and the Earth's rotation angle in ancient times. Periodic and abrupt changes in the Earth's rotation rate in ancient times are confirmed (Kawabata et al. 2004, Sôma et al. 2003, 2004a, 2005, Tanikawa \& Sôma 2004). The time systems in ancient China and Japan are investigated to analyze the ancient records of astronomical phenomena (Sôma et al. 2004b).

ROMANIA. Popescu at Bucarest reports that a CCD Apogee 47p camera has been installed at the $38 / 600 \mathrm{~cm}$ Prin-Merz Astrograph and will be used to image fields with ICRF sources.

RUSSIA. Devyatkin (St. Petersburg) reports on the fully-automated 32/320 cm Cassegrain telescope ZA-320 for positional and photometric CCD observations of solar system bodies as faint as $V=18$ (Devyatkin et al. 2004). The pointing and tracking of the Pulkovo $26^{\prime \prime}$ refractor has also been automated (Izmailov et al. 2004).

UKRAINE. Shulga at Nikolaev reports that the $30 / 150 \mathrm{~cm}$ Fast Robotic Telescope made at Nikolaev Astronomical Observatory in 2003 was put into operation. Its CCD camera can be used in three different modes: a guided stare mode, drift-scan, and satellite tracking. A method based on B-splines to match the CCD images has been developed.

Kleshchonok at Kiev reports that a portable TV-based complex CCTV for the observations of stellar occultation by the Moon was completed in 2003. So far 45 stellar occultations have been observed.

USA. van Altena (New Haven, CT) reports on the astrometric performance of the OPTIC camera, which uses the new orthogonal-transfer CCDs and is installed at WIYN $3.5 \mathrm{~m}$ telescope. In 5-min exposures the image-centering precision is as good as 2 mas on the images with $\mathrm{FWHM}=0.6^{\prime \prime}$.

Design studies were performed by Zacharias (Washington) in collaboration with Laux (Tautenburg) and EOS Technologies for the USNO Robotic Astrometric Telescope (URAT), a $0.85 \mathrm{~m}$ aperture and $4.5^{\circ}$-FOV instrument for a planned all-sky survey down to $\mathrm{R}=20^{\mathrm{m}}$ with a 5 to 10 mas accuracy.

\section{Extragalactic Reference Frame, Theory}

BRAZIL. Andrei at Rio de Janeiro reports on the continuing work on the ICRF optical counterparts. Fienga and Andrei (2004), Assafin et al. (2003, 2005) obtained optical positions for ICRF sources while Camargo et al. (2005) determined the near-infrared positions of ICRF sources. Silva Neto et al. (2002) proposed a morphological explanation for the observed pattern in the 'radio-optical' positional differences. Fienga and Andrei (2002) and Silva Neto et al. (2005) analyzed the synergy of ICRF and modern astrometric catalogues. Camargo et al. (2003) extended the ICRF to 16th magnitude in special regions using observations with Bordeaux and Valinhos meridian circles.

CHINA PR. Tang at Shanghai reports that new optical positions for 80 radio sources $\left(\sigma=0.1^{\prime \prime}\right)$ were obtained using improved reductions, better reference catalogues, and re-calibrated images from 350 plates observed at Torino Observatory. Collaborative work between SHAO and Torino, such as the analysis of systematic errors in the GSC-II catalogue, etc. has been carried out.

Ren at Nanjing reports that two two-epoch expressions (series) of precession quantities for both the IAU2000A and the post-IAU 2000 precession-nutation models were obtained. Applied to coordinate transformations within the period from 1800 to 2200, the precision of precession expressions based on the IAU2000A is $\sim 1$ mas, whereas in the case of postIAU 2000 model, the precision can reach $\sim 1 \mu$ as. These expressions have been adopted in calculations for the Chinese Astronomical Ephemeris 2005 (Ren et al. 2005). 
FRANCE. Kovalevsky at Cote d'Azur reports on writing the book "Fundamentals of Astrometry" (Kovalevsky \& Seidelmann 2004) and updating the book "Modern Astrometry" (Kovalevsky 2002) and its translation into Russian. He also studied the aberration effect in the proper motions of stars.

Paris Observatory reports on the process of maintenance and extension of the ICRF via examination and validation of individual reference frames contributed by various international organizations. In 2003-2005, the reference frames obtained at VLBI have been studied and validated by comparison with the ICRF and the ICRF-Ext.1 (IERS Annual Report 2003, 2004, 2005). As the result, the global orientation of these frames w.r.t. ICRS were derived at a sub-mas level. Individual frames show no significant deformations. Two extensions of the ICRF have been added since its definition: ICRF-Ext.1 (1998 IERS Annual Report) and ICRF-Ext.2 (Fey et al. 2004). These two extensions augment the ICRF by 109 new sources, conserving the list and the coordinates of defining sources.

Feissel-Vernier (Paris) reports on the stability studies of ICRF radio sources observed with VLBI. A selection of stable sources improves the stability of the resulting reference frame (Feissel-Vernier 2003, Arias \& Bouquillon, 2003). It is estimated that with only 250 stable sources the axes of ICRF can be maintained with an accuracy of $5 \mu$ as along with a $30 \mu$ as precision in the source positions. The determination of precession and long-term nutations is shown to be sensitive to the source selection at the level of $40 \mu \mathrm{a} \mathrm{yr}^{-1} \mathrm{~s}$ and $20 \mu \mathrm{as} \mathrm{yr}^{-1}$, respectively, and that of UT1-at the level of $0.2 \mu \mathrm{s} \mathrm{yr}^{-1}$ (Feissel-Vernier et al. 2005).

Souchey (Paris) reports that the number of recorded optical quasars steadily grows, reaching 48921 QSOs in the Véron-Cetty and Véron (2003) compilation. About $67 \%$ of ICRF sources are also found in this compilation (IERS Annual Report 2003), thus providing a source of photometric \& astrometric data.

Souchey \& Cognard report on pulsar timing observations in 2004 at the Nançay Radio Telescope with the aim to establish a link between the reference system of pulsars and the dynamical reference frame materialized by the ecliptic. The critical part in this link is the accuracy of the solar system body ephemeris.

ROMANIA. Popescu at Bucarest reports that CCD observations of ICRF sources down to $V=21$ have been obtained in 101 fields at the $60 \mathrm{~cm}$ Zeiss telescope in Belogradchik, Bulgaria under regional collaboration. Various dense catalogues were used to reduce the 6.1x6.1-arcmin-size CCD fields.

RUSSIA. Kumkova (St. Petersburg) reports on the efforts of ICRS realization in radio and optical wavelengths in the framework of the IAU 2000 Resolutions and transformations between BCRS \& GCRS (Kumkova et al. 2004).

Bobylev reports on a 0.4 mas $\mathrm{yr}^{-1}$ spin in HCRF with respect to the independent realizations of inertial reference frames (Bobylev 2004).

UK. van Leeuwen (Cambridge) reports on the re-analysis of the Hipparcos data summarized in van Leeuwen (2005) and van Leeuwen \& Fantino (2005). This study aimed at a better understanding of the dynamics of the satellite and of the methods used to derive astrometric parameters from the Hipparcos data. Firstly, the study resulted in the discovery of large numbers $(\sim 1,500)$ of scan-phase jumps caused by the non-rigidity of the spacecraft and the presence of $\sim 150$ minor hits. Both of these effects were known to exist in the published data, but had been underestimated in their influence on the data. The result for the published data was a considerable non-Gaussian noise component in the abscissa residuals, with strong, and varying, correlations between errors of stars separated by $n$ times the basic angle, where $n=0,1, \ldots, 9$. This in turn created serious problems in deriving star cluster parallaxes, for which uncertain correlations had to be taken into account to obtain reliable formal error estimates. 
Secondly, the Global Iterative Solution was studied in detail. It deals with reconstruction of the along-scan attitude and determination of the astrometric parameters. For this process to operate properly and produce ultimately absolute parallaxes, it is essential that data from both fields of view of the instrument contribute significantly to the attitude reconstruction. This causes problems when a field is mainly populated by bright stars. In such a case the weight of such a field has to be artificially reduced. Experiments with this iterative process and the weight reductions, show that this is a very slowly converging iteration, with a long-lasting "memory" of the starting conditions.

UKRAINE. Pinigin at Nikolaev reports that the joint project "Linking Optical-Radio Reference Frames" initiated by five observatories in China, Turkey, Russia and Ukraine is completed. The results of this project are presented in the form of a catalogue of positions for 200 extragalactic radio sources in the declination zone $-40^{\circ}<\delta<+80^{\circ}$. Among them, 140 are on the system of UCAC2 reference stars. The components of the global orientation angle between the optical and radio reference systems, determined at a 5 mas accuracy level, are as follows: $\varepsilon_{x}=-1.1 \pm 5.1, \varepsilon_{y}=-3.9 \pm 4.9, \varepsilon_{z}=8.1 \pm 4.4$ mas.

Kislyuk and Kazantseva at Kiev report that more than 25,000 observations of occultations and grazing occultations of the stars, planets and their satellites by the Moon have been collected. The lunar occultation database includes more than 10,000 stars down to 13th magnitude accumulated in 1963-2003 at 79 sites in the FSU. This database was analyzed for the spin vector in the HCRS, as realized by the Hipparcos Catalogue. The following spin components were found: $\omega_{x}=+1.20 \pm 0.65, \omega_{y}=+0.11 \pm 0.15, \omega_{z}=-3.20 \pm 2.20$ mas $\mathrm{yr}^{-1}$.

Fedorov at Kharkov reports that a new astrometric catalogue XC1 of faint reference stars has been made at the Kharkiv National University. The mean positions and proper motions of stars are derived from a combination of POSS-1, POSS-2 and 2MASS data. The XC1 extends the system of HCRS to mag $=19^{m}$ within one square degree around the ICRF sources in the northern hemisphere and contains $\sim 1.3$ million stars. At the mean epoch, the internal errors of XC1 range from 40 to 120 mas for positions and from 2 to

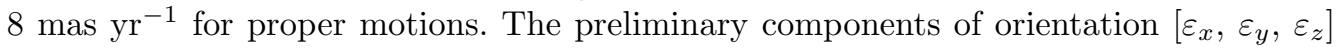
and spin $\left[\omega_{x}, \omega_{y}, \omega_{z}\right]$ are $[-1 \pm 10,-13 \pm 10,+4 \pm 10]$ mas and $[-0.03 \pm 0.34 ;-0.35 \pm 0.31$; $-0.01 \pm 0.33]$ mas $\mathrm{yr}^{-1}$, respectively.

Pinigin at Nikolaev reports that a new version of the AMC2003 catalogue was derived using the observations with the $18 / 248 \mathrm{~cm}$ Axial Meridian Circle in 2003. The catalogue contains 10,900 stars $(V=8-15)$ in the ecliptic zone near extragalactic radio sources. The accuracies range between $0.05-0.06^{\prime \prime}$ for magnitude $9-11$, and $0.08-0.12^{\prime \prime}$ for magnitude 14-15 stars. The catalogue was made in cooperation with Pulkovo Observatory (Russia).

USA. Kaplan (Washington) has prepared USNO Circular 179 which contains a comprehensive description of the IAU 1997 and 2000 resolutions on positional astronomy and their implementation.

Urban (Washington) chairs the Commission 8 WG "Densification of the Optical Reference Frame". Zacharias and collaborators continued working on the radio-optical reference frame link by having obtained deep optical observations of over 500 compact extragalactic sources at KPNO and CTIO.

Platais (Baltimore, MD) reports on the Deep Astrometric Standard (DAS) initiative. Meeting the growing need to calibrate the focal plane arrays of very large telescopes, it is vital to set up a few deep $(V=25)$ astrometric standards at the precision level of 5-10 mas, using a variety of telescopes and techniques (see astro/ph-0509606). A broad international collaboration is called upon to accomplish this project. 


\section{Positions and Proper Motions}

ARGENTINA. Carlos Lopez at San Juan reports that $\sim 80 \%$ of the planned observations at $-50^{\circ}<\delta<+30^{\circ}$ with the San Fernando Automated Meridian Circle have been completed. To study Earth rotation, the polar motion and latitude variations, a subset of Hipparcos stars at $-60^{\circ}<\delta<-3^{\circ}$ is regularly observed with the Photoelectric Astrolabe II. The collection of photographic plates at Cordoba and La Plata observatories has been re-structured and partially digitized.

CHINA PR. Jin at Shanghai reports that 13 astrometric standard fields for LAMOST (Large Sky Area Multi-object Fiber Spectroscopic Telescope) were selected in the areas with open clusters and star fields near Gould's Belt. The observations are being carried out with the $60 / 90 \mathrm{~cm}$ Schmidt telescope at Xing Long station of the National Astronomical Observatory, Beijing (Jin et al., 2005).

Using 653,790 observations of stars with the photoelectric astrolabe at San Juan Observatory, Argentina, a Third San Juan Photoelectric Astrolabe Catalogue (CPASJ3) was constructed. The comparisons between CPASJ3 and the Hipparcos Catalogue produced corrections which were applied to the CPASJ3. The catalogue also contains 69 radio stars (Lu et al. 2005).

BELGIUM. Pourbaix at Brussels reports on a collaborative project with Knapp and Ivezic from Princeton University on identification of over 300 candidate binaries in SDSS (Pourbaix et al. 2004) using a filter-dependent positional shift, also known as ColorInduced Displacement of binaries.

FRANCE. Ducourant (Bordeaux) reports on the first all-sky proper motion catalogue of 1250 pre-main sequence stars (Ducourant et al. 2005). The reductions of the Bordeaux $\mathrm{CdC}$ zone have resulted in the CdC2000 catalogue of 344,781 stars (Rapaport et al. 2005) and is free of the Tycho-2 propagated systematic errors. A new proper motion catalogue PM2000 of 2.7 million stars has been derived by combining CdC measurements and the Bordeaux Meridian Circle catalogue M2000 (Ducourant et al. 2005).

Fresneau at Strasbourg reports on new positions and proper motions in the Coalsack regions as part of a collaborative programme in science between France and Australia. Stellar orbits deflected by the mass of the dark clouds have been analyzed (Fresneau et al. 2003).

GERMANY. Schwan at Heidelberg reports on the continuing work on ARIGFH astrometric database, now comprising $\sim 10$ million observations from 1,400 catalogues, all transformed onto the HCRS.

RUSSIA. Khrutskaya (St. Petersburg) reports on the Pul-3 catalogue of positions and absolute proper motions of 58,483 stars, derived from the plates of the Pulkovo Normal Astrograph (Khrutskaya et al. 2004).

SPAIN. Muiños at San Fernando reports that the first catalogue of positions obtained with the San Fernando Automated Meridian Circle in San Juan (Argentina) contains 6.5 million stars $(V<16)$ at $-15^{\circ}<\delta<+3^{\circ}$. The Real Instituto y Observatorio de la Armada (ROA) is soon expected to be the sole operator of the Carlsberg Meridian Telescope on La Palma.

Nuñes and Muiños report on the continuing work on automation of the Baker-Nunn camera to be installed at Montsec Observatory (details in the 1999-2002 Report).

Digitization of the $\mathrm{CdC}$ and $\mathrm{AC}$ plates from the $-9^{\circ}<\delta<-3^{\circ}$ zone has been completed at ROA and the CD-ROMs of the scans are available.

UK. In April 2003, reports Evans (Cambridge), CMC13 was released covering the primary survey area of $-3^{\circ}<\delta<+30^{\circ}$. Observations by the Carlsberg Meridian Telescope have been carried out to extend the area to $-30^{\circ}<\delta<+50^{\circ}$ and work on producing 
an extended catalogue, $\mathrm{CMC} 14$, is underway. It is estimated that the external accuracy for $\mathrm{CMC} 14$ is 30 mas in right ascension and declination and 0.025 magnitudes in $r^{\prime}$ for the bright end. The magnitude limit of the catalogue is $r^{\prime}=17$.

Argyle (Cambridge) reports that in 2003 a series of 650 plates, taken in 1892-1930 for the Sydney Observatory Astrographic Programme along the 4th galactic quadrant, was scanned by the APM and used in combination with second epoch positions from Quick V Schmidt survey plates to produce proper motions for a sample of stars along the Sagittarius spiral arm (Fresneau et al. 2005).

WFCAM, a near-IR wide-field camera for UKIRT, has been commissioned in spring 2005, reports Irwin (Cambridge). Relative astrometry of better than 10 mas accuracy can be achieved at the bright end. A substantial fraction of the observing with this instrument will be for the UKIDSS surveys, including proper motions.

UKRAINE. Yatsenko at Kiev reports that a 2.0 version of the FONAC catalogue of positions, proper motions, and BVR photometry for two million stars has been released. All 1678 plates of the FON program were re-reduced using Tycho-2, resulting in a 19 mas precision for positions, and a 3.7 mas $\mathrm{yr}^{-1}$ uncertainty in proper motions.

Sergeeva at Kiev reports that a catalogue of 25,900 wide-field plates of the MAO NASU Plate Archive was prepared in a computer-readable form and integrated into the international Wide Field Plate Database (WFPDB).

Fedorov at Kharkov reports that a differential catalogue of right ascensions $(\sigma=$ 200 mas) for 1043 stars from PFKSZ, Fabricius and Lazorenko lists was made, using the 1982-1985 observations with the Repsold meridian circle.

USA. Zacharias (Washington DC) reports on the second release of the US Naval Observatory CCD Astrograph Catalog (UCAC2), containing accurate positions and proper motions of $48,330,571$ stars down to $R=16$ and covering the $-90^{\circ}<\delta<+40^{\circ}\left(52^{\circ}\right)$ sky (Zacharias et al. 2004). The UCAC2 proper motions are derived by including the AC2000 and over 100 ground-based catalogues as well as Hipparcos and Tycho-2 data and unpublished measures of NPM and SPM plates by D. Monet.

Monet (Flagstaff, AZ) reports that the scanning of all applicable Schmidt survey plates was completed and the all-sky deep USNO-B1 catalogue of over a billion stars was constructed, including proper motions (Monet et al. 2003). Zacharias reports that $\sim 1,900$ AGK2 plates were measured with the USNO StarScan and scanning of the Hamburg Zone and the USNO astrographs plates $(n \sim 3,500)$ is continuing.

The Naval Observatory Merged Dataset (NOMAD) was created containing the best astrometric data for any given star, mainly from Hipparcos, Tycho-2, UCAC2 and USNO-B1 catalogues.

Stone continued operation of the Flagstaff Astrometric Scanning Transit Telescope (FASTT) at the rate of about 41,000 CCD frames per year to support research on minor planet (MP) ephemerides, MP masses, MP occultation predictions, astrometric calibration regions, space missions, celestial reference frames and others. Single measurement errors are in the 47-300 mas range for $V=3.5-17.5$.

Hanson, Klemola, and Jones (Santa Cruz, CA) completed the Lick Northern Proper Motion (NPM) program after a half-century of work by 3 generations of Lick Observatory astronomers (Hanson et al. 2004). The two NPM Catalogues (NPM1 = CDS I/199A; NPM2 = CDS I/283A) provide absolute proper motions, accurate positions, and photometry for 378,360 stars from $8<B<18$, covering the northern $2 / 3$ of the sky $\left(\delta>-23^{\circ}\right)$.

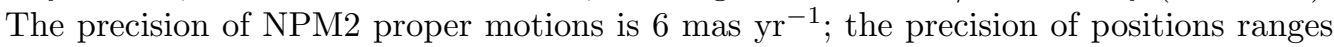
80-200 mas, depending on epoch. Klemola et al. (2004) compiled the NPM2 Cross- Identifications and Appendices (CDS I/293), with 46,887 entries listing star names, stellar 
type classifications, and publication references for 34,868 "special stars" of astrophysical interest.

van Altena (New Haven, CT) reports on the SPM3 catalogue (Girard et al. 2004) providing absolute proper motions, positions, and $B V$ photometry for 10.7 million objects, down to $V=17.5$. The catalogue covers an irregular area of 3700 sq. degrees at $-45^{\circ}<\delta<-20^{\circ}$. Observations were made with the $51 \mathrm{~cm}$ double astrograph in El Leoncito (Argentina). The catalogue is based on plate scans obtained by D. Monet using the USNO Precision Measuring Machine.

van Altena also reports on the schedule and activities to complete the SPM second epoch by CCD observations. With funding from the Argentine CONICET, a new astrometric-grade CCD camera was installed for the blue lens of the $51 \mathrm{~cm}$ double astrograph. Much of the efforts are spent on understanding the nature of systematic errors in the CCD data.

Hartkopf (Washington) reports on a catalogue of rectilinear elements for double stars yielding more precise differential proper motions. Since mid-2002 nearly 4,200 speckle interferometric observations of visual binaries have been produced at the USNO $26^{\prime \prime}$ telescope.

VENEZUELA. Abad at Merida reports on studies of systematic patterns in the stellar proper motions using the generalized Herschel's method of great circles in polar representation (Abad et al. 2003).

\section{Trigonometric Parallaxes, Nearby Low-Mass Star}

BELGIUM. Pourbaix at Brussels and Platais at Washington report on the effort to clarify the status of late variable stars classed in the Hipparcos Catalogue as VariabilityInduced Movers (Pourbaix et al. 2003, Platais et al. 2003). It turns out that revised chromaticity corrections applied to the Hipparcos IAD data considerably reduced the number of VIM solutions and in some cases resulted in substantially revised parallaxes.

GERMANY. Röser at Heidelberg reports on a new trigonometric parallax programme started with observations of the ten coolest known subdwarfs (sdM7.5-sdL) with Omega 2000 at the $3.5 \mathrm{~m}$ telescope at Calar Alto.

Scholz at Potsdam reports that the cross-identification of Luyten's high proper motion catalogues with the 2MASS led to the discovery of five new nearby red dwarfs with spectroscopic distances of less than 10 pc (McCaughrean, Scholz \& Lodieu 2002, Scholz et al. 2005). One of the nearest and brightest L dwarfs, SSSPM J0829-1309, has been detected in the SuperCOSMOS Sky Survey (SSS) data. Three other nearby L dwarfs were found by Lodieu, Scholz \& McCaughrean (2002). A number of objects with very large proper motions $(>1$ " $/$ yr) have been discovered using SSS and 2MASS data. Among them there are the nearest brown dwarf $\epsilon$ Indi B (Scholz et al. 2003) and other L and T type dwarfs (Scholz et al. 2004).

RUSSIA. Vityazev (St. Petersburg) reports on spectral parallaxes of 150 thousand Tycho-2 stars, allowing studies of kinematics of stars outside the range of the Hipparcos Catalogue stars.

USA. Using data from HST Fine Guidance Sensors Benedict \& McArthur (Austin, TX) and collaborators have obtained precise parallaxes $(\sigma=0.3 \mathrm{mas})$ for: five cataclysmic variables (V1223 Sgr, WZ Sge, YZ Cnc, RU Peg, and EX Hyd); $\delta$ Cep; the Pleiades; the central star of NGC 6853; GJ876 and 55 Cnc.

The USNO Flagstaff Station (NOFS) continued its parallax programme at the $1.55 \mathrm{~m}$ Strand telescope. 
Gatewood (Pittsburgh, PA) reports on the continuing parallax programme at Allegheny Observatory with the MAP system and CCD observing, also using the 100 inch DuPont telescope in Chile. With a $\sim 4$-yr epoch span 1 mas errors are achieved, operating over a wide magnitude range from naked eye (Gatewood 2005) to 18th magnitude.

\section{Special Projects}

\subsection{Solar System}

BRAZIL. Veiga, Vieira Martins, Rapaport, Teixeira and collaborators report on astrometric observations of Saturn, Uranus, Neptune, Pluto and some of their satellites at Valinhos and Bordeaux (France), summarized in several publications.

Andrei from Rio de Janeiro reports on the solar diameter observations and analysis by Penna et al. (2002), Reis Neto et al. (2003), Emilio \& Leister (2005), Kuhn et al. (2004). Over the period of major solar outbursts the multi-site solar diameter observations with the CCD astrolabe were analysed by Andrei et al. (2004).

CHINA PR. Jin at Shanghai reports that two image-processing techniques were developed to measure precisely the positions of a primary planet (Jupiter or Saturn) and its satellites. One of them uses the edge of the planetary image and fits it with an ellipse. The other is used to remove the positional effect of scattered light by a planet on the measurements of a close satellite. CCD images of Jupiter and its Galilean satellites show that a $0.03^{\prime \prime}$ precision can be reached (Peng et al. 2003a). For Saturn and its major satellites (S3-S6), about $0.06^{\prime \prime}$ can be reached (Peng et al. 2003b, 2005). The analytical TASS theory was used for astrometric calibrations of the CCD frames and theoretical computations. The observations of Tethys, Dione, Rhea and Iapetus have a standard deviation of $\sim 0.06^{\prime \prime}$ while for a few of the inner satellites, 2-3 times poorer residuals were obtained (Qiao et al. 2004).

The photographic and CCD observations of Phoebe at SHAO, Yunnan and Bordeaux observatories from 1904 to 2003 were reduced into the system of UCAC2 and used to obtain a new orbit. The residuals derived from this orbit are significantly smaller than that of the orbit computed by Arlot, using old photographic observations only (Shen et al. 2005).

FRANCE. Bordeaux CCD Meridian Circle observations of Pluto and Saturn were compared to DE403 and DE405 ephemerides, pointing out large 100 mas differences (Rapaport et al. 2002).

RUSSIA. Devyatkin (St. Petersburg) reports on new observations of small satellites of Jupiter and Saturn (Devyatkin et al. 2002) obtained at ZA-320. Sets of positional data were obtained with the Pulkovo $26^{\prime \prime}$ refractor for Saturn and its satellites (Kiseleva et a. 2002, 2004).

UKRAINE. Hudkova at Nikolaev reports that 579 CCD observations of 24 minor planets were obtained at the $12 / 205 \mathrm{~cm}$ Multi-channel Telescope and reported to MPC. In 2004-2005, minor planets and NEAs were observed with the $150 \mathrm{~cm}$ Russian-Turkish telescope RTT-150 in collaboration with Kazan State University and the National Observatory of Turkey. In total, 2455 positions ( $\sigma=50$ mas) of 39 minor planets brighter than 19th magnitude and 207 positions of eight NEAs down to 21.5 magnitude were obtained.

In 2002-2003, a number of Galilean satellite mutual events (9 occultations \& 14 eclipses) were observed with the CCTV camera (see Sect. 2) under the aegis of IMCCE (France). 
Photographical positions of Venus (408), Mars (408), Uranus (218), and Neptune (217) observed 1961-1998 were reduced into the system of ICRS and incorporated into the international databases of planetary data. Positions of Uranus and Neptune were used by M.Standish at JPL for the construction of the DE410 ephemeris.

Kleshchonok at Kiev reports on the solar disk radius measurements at the effective wavelength of $0.69 \mu \mathrm{m}$ from six observational sites in Romania during the August 11, 1999 Solar Eclipse. A careful analysis of the observed light curves produced a mean radius equal to $959.66 \pm 0.03^{\prime \prime}$.

Fedorov at Kharkov reports on the CCD observations of comets C/1999 S4, C/2001 A2, C/2002 X5, 2P at the AZT-8 telescope, processed using USNO-A2.0 and UCAC2 as reference catalogues. The calculated $(\mathrm{O}-\mathrm{C})$ values are correlated with the brightness variations of the comet's nucleus and its integral brightness.

USA. Pascu (Washington) reports on a 26-yr database of major planet satellite observations at USNO and an effort to re-measure the plate archive with planetary satellites at IMCCE (France).

\subsection{Open \& Globular Clusters \& the Galaxy}

CHINA PR. Chen at Shanghai reports that the proper motion of the globular cluster NGC 6656 (M22) was measured with respect to the background bulge stars using 12 frames, taken with the WFPC2 of HST at three epochs. The central velocity dispersion in radial and tangential components of the internal motion of cluster is $16.99 \mathrm{~km} \mathrm{~s}^{-1}$. The derived mass-to-light ratio $\mathrm{M} / \mathrm{L}_{v}=3.3 \pm 0.2$ is higher than the previous results (Chen et al. 2004).

Positions and absolute proper motions for 264 stars in the globular cluster NGC 6205 (M13) and of 534 stars for NGC 5272 (M3) were determined. The Galactic globular cluster M79 has also been studied astrometrically. From absolute proper motions, distances, and radial velocity data the space motions and orbits of all three clusters are derived and analysed (Chen et al. 2002, Wu et al. 2002, Wang et al. 2005).

Two new open cluster catalogues have been compiled. One of them, named CAT2, contains 144 clusters having absolute proper motion and radial velocity data. From CAT2 the 3-D velocities for each cluster relative to the Galactic Standard of Rest were derived. The statistical analysis shows that the spatial distribution of young open clusters is symmetric with respect to the sun and has a low scale-height and small velocity dispersion, while it is almost opposite for the old open clusters (Chen et al. 2003).

RUSSIA. Kanaev (St. Petersburg) reports on the proper motions in M67, NGC 7243, and NGC 7654 from the observations with MAGIS and Normal Astrograph, spanning over 100 yrs.

Vityazev (St. Petersburg) reports on a new method based upon wavelet analysis to identify inhomogeneities in the spatial distribution of stars. More than 200 statistically significant groups have been detected in the Tycho-2 data (Vityazev et al. 2004, Kazakevich et al. 2002).

UKRAINE, RUSSIA \& GERMANY. Kharchenko at Kiev, Schilbach at Heidelberg, and Piskunov at Moscow report on a comprehensive kinematic and photometric study of 520 open clusters, based upon the ASCC-2.5 catalog. The catalogue of astrophysical data (Kharchenko et al. 2005), the atlas of various diagrams, and the datasets of open cluster characteristics are valuable new sources of tangential (absolute proper motions) and radial velocities, spatial extent, distance, reddening, and age.

The same data on open clusters have served to study the parameters of the Galactic disk such as the constants of Galactic rotation, parameters of the velocity ellipsoid, elements of Galactic orbits, the scale-height of the disk and the Sun's z-distance. The 
new estimate of the total number of open clusters in the Milky Way is $\sim 63,000$. It appears that less than $\sim 10 \%$ of the Galactic disk population may have ever passed the phase of cluster membership.

Rybka at Kiev reports that a compilation catalogue (Rybka 2003) of MK spectral classification, astrometric and photometric data for $\sim 170,000$ stars is created to study kinematics of the Galaxy and its components.

USA. Cudworth (Yerkes Observatory) continued photographic astrometry work in selected open and globular clusters. Jones (Santa Cruz, CA) reports that second epoch observations on photographic plates with the Lick $36^{\prime \prime}$ telescope and additional CCD imaging with other Lick telescopes are completed. These observations will serve to determine proper motions in open clusters. Platais (Baltimore, MD) reports on deep astrometry in the WIYN Open Cluster Study (WOCS) programme. It has been proved that a combination of CCD mosaic images and old photographic plates from a $4 \mathrm{~m}$ unastrometric telescope can provide precise proper motions $\left(\sigma=0.15 \mathrm{mas} \mathrm{yr}^{-1}\right)$, as shown for the open cluster NGC 188 (Platais et al. 2003).

van Altena (New Haven, CT) reports on high-precision proper motions derived in the globular cluster NGC 6752 using the HST data (Drukier et al. 2003). An effort is also being made to use image deconvolution methods to recover for astrometry the HST WFPC exposures taken when the camera was seriously affected with spherical aberration.

Dinescu (New Haven, CT) reports on various projects on globular clusters and nearby dwarf spheroidal galaxies. Absolute proper motions of four globular clusters located in the inner Galaxy (bulge) were measured using the SPM plate material (Dinescu et al. 2003). This brings the total number of globular clusters measured with SPM material to 19. The programme continues for another ten clusters to be measured using the SPM CCD data as second epochs. The absolute proper motion of the Fornax dwarf spheroidal

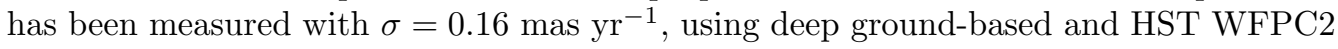
data. This is the most distant Milky Way satellite to have its proper motion measured to this precision. Proper motions of the more nearby dwarf spheroids, Sgr and CMa dwarf galaxies, have been determined based on the SPM3 data (Dinescu et al. 2005a) and the SPM plate material \& CCD data (Dinescu et al. 2005b), respectively.

van Altena (New Haven, CT) reports that kinematical properties of the Milky Way's thick disk have been studied using the SPM3 proper motions and 2MASS photometry. It appears that the thick disk has a considerable rotational velocity shear in the direction perpendicular to the Galactic disk. Korchagin et al. (2003) estimated the density of gravitating matter in the Galactic disk using Hipparcos proper motions and the BarbierBrossat \& Figon radial velocities catalogue. The estimated total mass density is close to the observed density of baryonic matter in the solar neighborhood.

\section{Space Astrometry}

NETHERLANDS. Perryman at Noordwijk reports on the European Space Agency's mission Gaia. Proposed in 1994 as a follow-up to the Hipparcos mission, scientific and technical studies led to the inclusion of the Gaia space astrometry mission within ESA's science programme in 2000. Since that time, numerous and comprehensive scientific and technical studies have been conducted within ESA, European industry, and the European scientific community. These have led to an iterative convergence of the scientific goals, as constrained by practical issues of technical feasibility, cost, and schedule. A milestone was reached in June 2005 with the completion of the industrial feasibility and development studies, and the release of ESA's industrial Invitation to Tender for Phases B2/C/D (detailed design, development, launch). The current target launch date is late 2011. 
Gaia is now expected to provide an all-sky survey, complete to 20 mag, and employing on-board object detection to observe more than 1 billion stars (and solar system objects to similar completeness limits). The full mission (5-year) mean-sky parallax accuracies are expected to be around 7 microarcsec at $V=10 ; 12-25 \mu$ as at $V=15$ (depending on spectral type); and 100-300 $\mu$ as at $V=20$. Comprehensive multi-epoch, multi-color photometry (of order 15 bands covering the optical wavelength range) will reach the same completeness limit. Radial velocity completeness will range between $V=13-18.5$ depending on spectral type, with accuracies of around $1-15 \mathrm{~km} \mathrm{~s}^{-1}$.

The ESA www site (http://www.rssd.esa.int/Gaia) maintains an up-to-date record of overall status, technical progress, working group meetings, and other information.

\section{Education in Astrometry}

van Altena (New Haven, CT) reports on the workshop on Basic Astrometry Methods held at Yale University in July 2005. The goal of this workshop was to provide an intensive introduction to the basic methods needed to utilize facilities with advanced astrometric capabilities. A total of 46 students and researchers attended: 31 from US institutions and 15 from other countries. The workshop lecturers were drawn primarily from Yale personnel and was funded by grants from the US National Science Foundation and Yale University. The lectures are available on the Yale Astronomy Department's web site. Following the Yale workshop, the Michelson Summer Workshop, also an educational program, was held on the campus of Caltech with an emphasis on using astrometry to discover extra-solar planets.

\section{Symposia, Colloquia, Conferences}

-Second Latin-American Meeting on Astrometry (ADeLa-2002) held in Araraquara, Brazil, September 2-5, 2002.

-IAU Colloquium 189 "Astrophysical tides: the effects in the solar and exoplanetary systems" held in Nanjing China PR, September 16-20, 2002.

-IAU 25th GA, JD 16 "The International Celestial Reference System, Maintenance and Future Realizations" held in Sydney, Australia, July 22, 2003.

-Journées 2003 "Astrometry, Geodynamics and Solar System Dynamics from Milliarcseconds to Microarcseconds" held in St. Petersburg, Russia, September 22-25, 2003.

-IAU Colloquium 196 "Transit of Venus" held in Preston, UK, June 7-11, 2004.

-Symposium "The Three Dimensional Universe with Gaia" held in Paris-Meudon, France, October 4-7, 2004.

-Journées 2004 "Fundamental Astronomy: New Concepts and Models for High Accuracy Observations" held in Paris, France, October 4-7, 2004.

-Conference "Astrometry in the Age of the Next Generation of Large Telescopes" held in Flagstaff AZ, USA, October 18-20, 2004.

-Meeting "Astrometry with Small Telescopes" held in Bucarest, Romania, October 21$24,2004$.

-Third Latin-American Meeting on Astrometry (ADeLa-2004) dedicated to Jürgen Stock and held in Merida, Venezuela, November 22-24, 2004.

\section{Acknowledgements}

We thank the designated Representatives for their contribution to this Report. 